\title{
Confronting Dark Energy Models with Astrophysical Data: Non-Equilibrium vs. Conventional Cosmologies
}

\author{
John R. Ellis ${ }^{a}$, Nikolaos E. Mavromatos ${ }^{\mathrm{b}}$, \\ Vasiliki A. Mitsou ${ }^{c}$,Dimitri V. Nanopoulos d,e,f \\ a TH Division, Physics Department, CERN, CH-1211 Geneva 23, Switzerland \\ b Theoretical Physics, Physics Department, King's College London, \\ Strand WC2R 2LS, UK \\ ${ }^{\mathrm{c}}$ Instituto de Física Corpuscular (IFIC), CSIC - Universitat de València, \\ Edificio Institutos de Paterna, P.O. Box 22085, E-46071 Valencia, Spain \\ ${ }^{\mathrm{d}}$ George P. and Cynthia W. Mitchell Institute for Fundamental Physics, \\ Texas A\&M University, College Station, TX 77843, USA \\ e Astroparticle Physics Group, Houston Advanced Research Center (HARC), \\ Mitchell Campus, Woodlands, TX 77381, USA \\ ${ }^{\mathrm{f}}$ Academy of Athens, Division of Natural Sciences, 28 Panepistimiou Avenue, \\ Athens GR-10679, Greece
}

\begin{abstract}
We discuss fits of cosmological dark energy models to the available data on highredshift supernovae. We consider a conventional model with Cold Dark Matter and a cosmological constant $(\Lambda \mathrm{CDM})$, a model invoking super-horizon perturbations (SHCDM) and models based on Liouville strings in which dark energy is provided by a rolling dilaton field (Q-cosmology). We show that a complete treatment of Q-cosmology requires a careful discussion of non-equilibrium situations (off-shell effects). The two main high-redshift supernova data sets give compatible constraints on $\Lambda \mathrm{CDM}$ and the other models. We recover the well-known result that $\Lambda \mathrm{CDM}$ fits very well the combined supernova data sets, as does the super-horizon model. We discuss the model-dependent off-shell corrections to the Q-cosmology model that are relevant to the supernova data, and show that this model fits the data equally well. This analysis could be extended to other aspects of cosmological phenomenology, in particular to the CMB and Baryon Acoustic Oscillations, which have so far been treated using on-shell models.
\end{abstract}

Key words: Observational cosmology, Dark energy, Supernovae PACS: 98.80.Es, 95.36.+x, 98.65.Dx 


\section{Introduction}

There is a plethora of astrophysical evidence today, from supernovae measurements, the spectrum of fluctuations in the cosmic microwave background [1-4], baryon oscillations and other cosmological data, indicating that the expansion of the Universe is currently accelerating. The energy budget of the Universe seems to be dominated at the present epoch by a mysterious dark energy component, but the precise nature of this energy is still unknown. Many theoretical models provide possible explanations for the dark energy, ranging from a cosmological constant [5] to super-horizon perturbations [6] and time-varying quintessence scenarios [3], in which the dark energy is due to a smoothly varying (scalar) field which dominates cosmology in the present era, such as a time-dependent dilaton field [7].

The current astrophysical data are capable of placing severe constraints on the nature of the dark energy, whose equation of state may be determined by means of an appropriate global fit. Most of the analyses so far are based on effective four-dimensional Robertson-Walker Universes, which satisfy on-shell dynamical equations of motion of the Einstein-Friedman form. Even in modern approaches to brane cosmology, which are described by equations that deviate during early eras of the Universe from the standard Friedman equation (which is linear in the energy density), the underlying dynamics is assumed to be of classical equilibrium (on-shell) nature, in the sense that it satisfies a set of equations of motion derived from the appropriate minimization of an effective space-time Lagrangian.

However, cosmology may not be an entirely classical equilibrium situation [7]. The initial Big Bang or other catastrophic cosmic event, which led to the initial rapid expansion of the Universe, may have caused a significant departure from classical equilibrium dynamics in the early Universe, whose signatures may still be present at later epochs including the present era. Three of us (JE, NEM, DVN) have indeed proposed one specific model for the cosmological dark energy which is of this type, being associated with a rolling dilaton field that is a remnant of this non-equilibrium phase, described by a generic noncritical string theory [8-10]. We call this scenario 'Q-cosmology'.

The central purpose of this paper is to confront the present cosmological data on high-redshift supernovae with non-equilibrium cosmologies [7-10], in which the dark energy relaxes at different rates, and compare the results with the predictions of the conventional $\Lambda \mathrm{CDM}$ model [5]. We also comment on a model with super-horizon perturbations superposed on an underlying Einstein-Friedman-Robertson-Walker Universe ${ }^{1}$ [6].

As we explain in more detail below, care must be taken in interpreting the

1 Our approach to this model is strictly phenomenological: we do address any of its deeper theoretical issues. 
Q-cosmology scenario proposed in Refs. [8-10]. Since such a non-equilibrium, non-classical theory is not described by the equations of motion derived by extremizing an effective space-time Lagrangian, one must use a more general formalism to make predictions that can be confronted with the current data. The approach we favour is formulated in the context of string/brane theory $[11,12]$, the best candidate theory of quantum gravity to date. Our approach is based on non-critical (Liouville) strings [7, 13, 14], which offer a mathematically consistent way of incorporating time-dependent backgrounds in string theory.

The basic idea behind such non-critical Liouville strings is the following. Usually, in string perturbation theory, the target space dynamics is obtained from a stringy $\sigma$-model [11] that describes the propagation of strings in classical target-space background fields, including the space-time metric itself. Consistency of the theory requires conformal invariance on the world sheet, in which case the target-space physics is independent of the scale characterising the underlying two-dimensional dynamics. These conformal invariance conditions lead to a set of target-space equations for the various background fields, which correspond to the Einstein/matter equations derived from an appropriate low-energy effective action that is invariant under general coordinate transformations. Unfortunately, one cannot incorporate in this way time-dependent cosmological backgrounds in string theory, since, to low orders in a perturbative expansion in the Regge slope $\alpha^{\prime}$, the conformal invariance condition for the metric field would require a Ricci-flat target-space manifold, whereas a cosmological background necessarily has a non-vanishing Ricci tensor.

To remedy this defect, and thus be able to describe a time-dependent cosmological background in string theory, the authors of Ref. [7] suggested that a non-trivial rôle should be played by a time-dependent dilaton background. This approach leads to strings living in numbers of dimensions different from the customary critical number, and was in fact the first physical application of non-critical strings [13]. The approach of Ref. [7] was subsequently extended $[8-10,14]$ to incorporate off-shell quantum effects and non-conformal string backgrounds describing other non-equilibrium cosmological situations, including catastrophic cosmic events such as the collision of two brane worlds, etc.

In our discussion of such models in this paper, we first review briefly their main predictions. We then demonstrate the importance of off-shell corrections to the naive equations of motion of non-critical Liouville string cosmology, which cause us to modify the naive equations of motion of Q-cosmology. We then demonstrate that the available supernova data are compatible with such non-critical-string-based cosmologies [8, 10].

The structure of the article is as follows. In Section 2 we summarise the basic equations of the first set of models we study, namely $\Lambda \mathrm{CDM}$ and the superhorizon model. Then, in Section 3 we discuss the Liouville Q-cosmology model 
of Refs. [8-10]. In particular, we discuss technical aspects of Q-cosmology, placing the emphasis on the off-shell corrections and deriving the fitting formulae to be used in our analysis. This part may be omitted by the reader who is more interested in the confrontation of this and other models with the available high-redshift supernova data that we present in Section 4. We find that the $\Lambda$ CDM model fits the data very well, as does the super-horizon model. We also show that the off-shell corrections to the Q-cosmology enable this model to be reconciled with the supernova data on equal footing with $\Lambda$ CDM. ${ }^{2}$ Our results and the possible outlook are summarised in Section 5.

\section{Basic Formulae for the Phenomenological Analysis of On-Shell Dark Energy Models}

In this Section we review the basic formulae needed in the phenomenological analysis of observational constraints on the cosmological parameters derived from supernova data and (in the case of the $\Lambda$ CDM) also baryon acoustic oscillations, in the context of on-shell models (i.e., excluding the Q-cosmology model to be discussed later). The relevant dynamics of any such cosmological Robertson-Walker model may be lumped into the expression for the Hubble parameter $H(z)$ in terms of the various components of the energy density of the Universe, represented in units of the (present-day) critical density for a spatially-flat Universe, $\Omega_{i} \equiv \rho_{i}^{0} / \rho_{c}^{0}$, where $i=\mathrm{M}$ for matter, including dark matter, $i=\Lambda$ for constant dark energy, i.e., a cosmological constant $\Lambda$, etc. Later in this work we discuss fits to the on-shell $(\Lambda \mathrm{CDM}$ and superhorizon) and off-shell (Q-cosmology) models discussed in the current and the next Sections, respectively.

\subsection{Standard $\Lambda C D M$ Model [5]}

In this case, we consider a spatially flat Robertson-Walker Universe containing dust-like matter that can be described as an ideal fluid, and a cosmological constant $\Lambda>0$ corresponding to a constant equation of state of the form:

$$
p=w_{0} \rho
$$

where $p(\rho)$ is the pressure (energy density) and $w_{0}=-1$. The standard Einstein-Friedmann equations for such a Universe yield:

$$
H(z)=H_{0}\left(\Omega_{\mathrm{M}}(1+z)^{3}+\Omega_{\Lambda}(1+z)^{3\left(1+w_{0}\right)}\right)^{1 / 2}
$$

where $H_{0}$ is the present-day value of the Hubble constant.

$\overline{2}$ A more complete comparison of this model with data on the cosmic microwave background ( $\mathrm{CMB}$ ) and baryon acoustic oscillations (BAO) would require further formal developments that lie beyond the scope of this paper. 


\subsection{Super-Horizon Perturbation Model [6]}

In this model, the Universe is assumed to be filled with only non-relativistic matter, and there is no Dark Energy of any sort. The metric takes the form:

$$
d s^{2}=-d t^{2}+a^{2}(t) e^{-2 \Psi(\vec{x}, t)} \delta_{i j} d x^{i} d x^{j}
$$

where $\Psi(\vec{x}, t)$ denotes the gravitational potential. The authors of Ref. [6] considered perturbations $\Psi(\vec{x}, t)$ that correspond to conformal contraction or stretching of the three-dimensional Euclidean space, $\left\{x^{i}, i=1,2,3\right\}$. Splitting the potential $\Psi=\Psi_{\ell}+\Psi_{s}$ into contributions from long-wavelength (superhorizon) modes, $\Psi_{\ell}$, and short-wavelength (sub-horizon) modes, $\Psi_{s}$, and ignoring the latter within our Hubble volume, one obtains [6]:

$$
d s^{2} \simeq-d t^{2}+\bar{a}^{2}(t) \delta_{i j} d x^{i} d x^{j}, \quad \bar{a}(t)=a(t) e^{-\Psi_{\ell}(t)+\Psi_{\ell 0}}
$$

with the normalization $\bar{a}_{0}=a_{0}=1$.

The analysis of Ref. [6] used the appropriate Einstein's equations to demonstrate that, up to trivial rescaling of spatial coordinates and absorbing the time-independent part of $\Psi_{\ell}$, the time dependence of $\Psi_{\ell}(t)$ may be cast in the form:

$$
\Psi_{\ell}(t) \simeq a(t) \Psi_{\ell 0}
$$

One therefore finds that the expansion rate of the Universe, as observed by a local observer who is restricted to observe within our Hubble volume, is given by:

$$
H(z)=\bar{a}^{-1} \frac{d \bar{a}(t)}{d t}=\frac{\bar{H}_{0}}{1-\Psi_{\ell 0}}\left(a^{-3 / 2}-a^{-1 / 2} \Psi_{\ell 0}\right),
$$

where $(1+z)^{-1}=\bar{a}(t)$. Combining (4) and (5), one may extract the following relation between $a$ and $z$ :

$$
1+z=a^{-1} e^{(a-1) \Psi_{\ell 0}}
$$

This model predicts acceleration of the Universe at the current era:

$$
q=-1+\frac{3 / 2-a \Psi_{\ell 0} / 2}{\left(1-a \Psi_{\ell 0}\right)^{2}} .
$$

It can easily be shown by direct comparison with conventional models that the super-horizon perturbations mimic a dark energy model with equation of state

$$
w_{D E} \Omega_{D E}=\frac{2}{3}\left(q-\frac{1}{2}\right) .
$$

In order to evaluate the constraints imposed by astrophysical data on this super-horizon model, we use the following integral of the Hubble parameter 


$$
\begin{aligned}
& E(z) \equiv H(z) / H_{0} \text { over } z: \\
& \int_{0}^{z} \frac{d z}{E(z)}=\left(1-\Psi_{\ell 0}\right) \int_{a(z)}^{1} d a a^{-1 / 2} e^{(a-1) \Psi_{\ell 0}}
\end{aligned}
$$

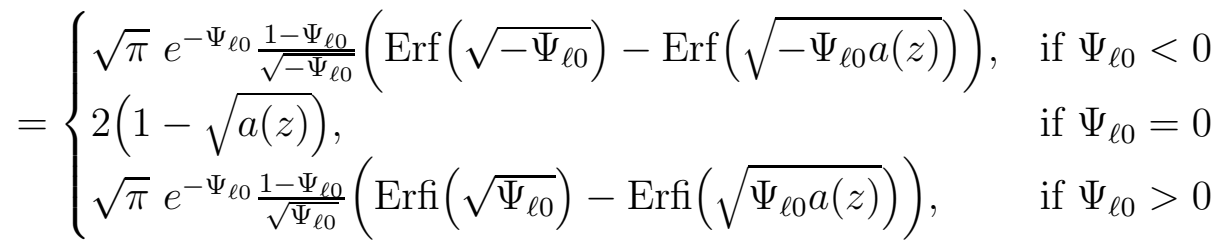

where Erf and Erfi are the standard and imaginary error function, respectively. We assume values $\left|\Psi_{\ell 0}\right|<1$, in order for perturbation theory to remain valid [6]. We reiterate that we do not address any of the deeper theoretical issues of this model.

\subsection{A Note on Relaxing Dark Energy Models}

There are many dark energy models in which the energy density relaxes in time. For example, relaxation at a rate proportional to $1 / t^{2}$, where $t$ is the cosmic time, may arise in certain non-critical string theory models [14]. In such a model, the rôle of the quintessence field is played by the dilaton field [7]. However, at least in the string theory context, a complete treatment of such a rolling dilaton field requires a non-trivial discussion of off-shell physics, which we present in the next section. For the moment, we neglect this complication (as might be valid for some non-string models of relaxing dark energy), and assume a naive on-shell physical framework in which the dark energy density relaxes according to a generic power law $1 / t^{n}$. Within this general framework, $n=0$ corresponds to a cosmological constant $\Lambda$ and $n=2$ corresponds to a naive interpretation of the Q-cosmology model of Ref. [8], in which only the conventional type of matter exists and the off-shell terms are not taken into account. Such a relaxing dark energy model would predict a Hubble parameter of the form:

$$
H(z)=H_{0}\left(\Omega_{\mathrm{M}}(1+z)^{3}+\Omega_{\phi}(1+z)^{n}\right)^{1 / 2},
$$

where $\Omega_{\phi}$ represents the present-day dilaton dark energy in the on-shell formulation. ${ }^{3}$ In such a model with $n=2$, the rolling dilaton dark energy would resemble a spatial curvature contribution, and such naive on-shell models are easily excluded on the same observational grounds that support experimentally the spatial flatness of the Universe. However, $n=0$ is certainly allowed, and it is important to know the permissible range of $n$. There is a formal correspondence between (11) and a generalized $\Lambda$ CDM model in which the vacuum energy $\Lambda$ obeys an equation of state with $w_{0} \neq-1(2)$, namely $n=3\left(1+w_{0}\right)$. However, the underlying physics may be entirely different. For models with

3 However, we emphasize again that this on-shell treatment is incorrect in the context of non-critical string theory, as we discuss below. 
relaxing dark energy (11), the standard data analysis shows that both the 'gold' and the SNLS dataset favour a value of the parameter $n$ appearing that is $\ll 2$. Indeed, the combined data prefer $n \sim 0\left(w_{0} \sim-1\right)$, i.e., a (nearly) constant vacuum energy, as in the $\Lambda \mathrm{CDM}$ model.

\section{Non-Critical Liouville String Q-Cosmologies}

In this Section we explore in more detail Q-cosmologies with relaxing vacuum energy in the context of Liouville strings, which provide a consistent treatment of off-shell, out-of-equilibrium effects. For the benefit of the reader, we review here the basic formalism and summarize the results, giving details of their derivation in the next two subsections, that may be skipped by a reader not interested in technical aspects. There are many specific cosmological models that may be formulated within the general framework of Liouville strings: see [8-10] for an extended discussion and references. Here we limit ourselves to

the main features of this cosmological framework, which do not depend on the details of the underlying microscopic string/brane models or compactification. We simply assume the theory to have been compactified (somehow) to a fourdimensional Robertson-Walker space-time, with a scale factor $a\left(t_{E}\right)$ in the so-called Einstein cosmic frame [7].

\subsection{General Formalism of Off-Shell Liouville Q-Cosmologies}

In the absence of matter, the Liouville-dressing approach of Ref. [14] and the (dynamical) identification of the Liouville zero mode with target time lead to generalized conformal invariance conditions for the fields of the gravitational multiplet of the string propagating in a four-dimensional background:

$$
\ddot{g}^{i}+Q \dot{g}^{i}=-\beta^{i}
$$

where the overdot denotes differentiation with respect to the world-sheet zero mode of the Liouville field $\phi$, the set of $g^{i}=\left\{G_{\mu \nu}, \phi, \ldots\right\}$ are string background fields (metric, dilaton, etc.), the $\beta^{i}$ are the respective world-sheet renormalization-group $\beta$-functions, and $Q$ is the square root of the centralcharge deficit that describes the departure from criticality. The negative sign in front of $\beta^{i}$ on the right-hand side of (12) corresponds to supercriticality for the string, in which case the central charge exceed its critical (conformallyinvariant) value. It is in this case that the Liouville mode has a time-like signature and can be identified with the target time [7, 14].

In critical strings, the vanishing of the $\beta^{i}$ would correspond to the ordinary Einstein/matter equations of the low-energy field theory derived from strings. The vanishing conditions correspond to an equilibrium situation. In noncritical string, however, these equations are replaced by (12), which express the restoration of conformal invariance by the Liouville mode. The physical reasons for an initial departure from conformal invariance might be a catastrophic 
cosmic event, such as the collision of two brane worlds or a Big Bang [8]. Such an event would cause a departure from equilibrium, which may not be inappropriate for the early era of the Universe. Following the identification of target time with the Liouville mode, the generalized conformal invariance equations (12) yield new dynamical equations that describe the non-equilibrium dynamics of the Liouville strings $[8-10,14]$.

If one further assumes that, after compactification to four space-time dimensions, one obtains a spatially-flat cosmological Robertson-Walker metric background, e.g., in the context of a brane model, the equations (12) define the dynamics of the resulting 'Liouville Q-cosmology', as we term such a noncritical string cosmology. The equations (12) are not the ordinary equations of motion corresponding to a four-dimensional gravitational effective action, but describe the dynamics of an off-shell relaxation process.

As explained in Ref. [8], the inclusion of matter does not change qualitatively this non-equilibrium, off-shell nature of such a model. Apart from the formal addition of extra terms in the gravitational $\beta$-functions, resulting from the coupling of matter to the gravitational field, which are needed for general coordinate invariance in the target-space, in most models of physical interest may be included in such a way that the main source of non-criticality is the gravitational and moduli sector, while the matter $\beta^{i}$ functions themselves are (almost) vanishing. In such a case, the matter fields feel the presence of the off-shell Liouville terms on the left-hand-side of (12) via their gravitational couplings.

The presence of these off-shell Liouville terms, which are exclusive to noncritical strings, results in a modification of the critical energy density condition for the Liouville cosmology, which ensures spatial flatness. This critical density is therefore different from that in conventional (on-shell) Friedman-RobertsonWalker (FRW) cosmologies.

The essential formalism is that of Ref. [7], in which all physically relevant quantities should be expressed in the Einstein frame, in terms of the Einstein cosmic time. The four-dimensional matter action (including radiation fields) couples to the dilaton field non-trivially, in a way that is specific to the various matter species, as a result of purely stringy properties of the effective action [11]. A generic $\sigma$-model-frame effective four-dimensional action with dilaton potential $V(\phi)$, which could even include higher string-loop corrections, has the form:

$$
\begin{aligned}
S^{(4)}= & \frac{1}{2 \alpha^{\prime}} \int d^{4} x \sqrt{-G}\left[e^{-\Psi(\phi)} R(G)+Z(\phi)(\nabla \phi)^{2}+2 \alpha^{\prime} V(\phi) \ldots\right]- \\
& \frac{1}{16 \pi} \int d^{4} x \sqrt{G} \frac{1}{\alpha(\phi)} F_{\mu \nu}^{2}-I_{\mathrm{m}}(\phi, G, \text { matter }),
\end{aligned}
$$

in the notation of Ref. [15], with the various factors $\Psi, Z, \alpha$ encoding informa- 
tion about higher string loop corrections. Also, $F_{\mu \nu}$ denotes the radiation field strength and $I_{\mathrm{m}}(\phi, G$, matter $)$ represents matter contributions, which couple to the dilaton $\phi$ in a manner dictated by string theory, with specific scaling laws [11] under shifts of the dilaton field $\phi \rightarrow \phi+$ const. In cases [8] where only the string tree level plays a rôle for late times, the various form factors simplify, e.g., $\Psi(\phi)=2 \phi, Z(\phi)=4$, etc. However, for reasons of generality, here we keep the form (13). As we shall see, there is sensitivity in the present data to terms in the dilaton potential with higher powers of the string coupling. When higher loop corrections are important, these factors have a complicated form, for instance one has $e^{\Psi(\phi)}=c_{0} e^{-2 \phi}+c_{1}+c_{2} e^{2 \phi}+\ldots$, with various constants $c_{i}$, with the powers of the square of the string coupling $g_{s}^{2}=e^{2 \phi}$ counting closed string loops, as appropriate for the gravitational multiplet. For simplicity, in the present work we ignore the antisymmetric-tensor four-dimensional field, which, as discussed in Ref. [7], corresponds to an axion field.

Higher loop corrections may result in a modified dilaton potential for the noncritical string theory of the form

$$
V=2 Q^{2} e^{2 \phi}+\tilde{V}
$$

where the central charge deficit $Q^{2}$ encodes the microscopic non-equilibrium physics $[8,9]$. In the models we are considering in this work, $Q^{2}>0$ and the string theory is supercritical [7].

Assuming a normal fluid form for matter or radiation, with stress tensor $T_{\mu}^{\nu E}=$ $\operatorname{diag}\left(-\rho, p \delta_{i}^{j}\right)$ in the Einstein frame [7], we obtain the following gravitational equations of motion in our case (we work here in units $M_{P}^{2}=1 / 8 \pi G_{N}=1$, where $M_{P}$ is the four-dimensional Planck constant) [10]:

$$
\begin{gathered}
3 H^{2}=\rho_{m}+\rho_{\phi}+\frac{e^{2 \phi}}{2} \mathcal{J}_{\phi}, \\
2 \frac{d H}{d t_{E}}=-\rho_{m}-\rho_{\phi}-p_{m}-p_{\phi}+a^{-2}\left(t_{E}\right) \mathcal{J}_{i i}, \quad i=1,2,3, \\
\rho_{\phi} \equiv \frac{1}{2}\left(2\left(\frac{d \phi}{d t_{E}}\right)^{2}+V(\phi)\right), \quad p_{\phi} \equiv \frac{1}{2}\left(2\left(\frac{d \phi}{d t_{E}}\right)^{2}-V(\phi)\right), \\
\frac{d^{2} \phi}{d t_{E}^{2}}+3 H \frac{d \phi}{d t_{E}}+\frac{1}{4} \frac{\partial V}{\partial \phi}+\frac{1}{2}\left(\rho_{m}-3 p_{m}\right)=-\frac{3 \mathcal{J}_{i i}}{2 a^{2}}-\frac{1}{2} e^{2 \phi} \mathcal{J}_{\phi},
\end{gathered}
$$

where $t_{E}$ is the Einstein-frame Robertson-Walker cosmic time [7] and the potential of the dilaton assumes the form (14), including loop corrections $\tilde{V}$ (14). We note that the equations (15) differ from those holding in on-shell cosmologies by the Liouville out-of-equilibrium contributions $\mathcal{J}$, which are exclusive to our treatment $[8,10,14]$.

The equations (15) need to be supplemented by the appropriate Curci-Paffuti equation [16] expressing the renormalisability of the $\sigma$-model fields $[9,10]$, 
which is also exclusive to our non-critical string approach. This equation relates the dilaton world-sheet $\beta$-function to the rest of the $\beta$-functions in the problem (for the graviton, etc.), and essentially expresses the (time-dependent) central charge deficit in terms of the physical fields in the problem,

$$
\frac{d \mathcal{J}_{\phi}}{d t_{E}}=-6 e^{-2 \phi}(H+\dot{\phi}) \frac{\mathcal{J}_{i i}}{a^{2}}+\ldots
$$

where the overdot henceforward denotes a derivative with respect to the cosmic time $t_{E}$, the '...' indicate terms due to higher order in string loops, and the values of $\mathcal{J}_{\phi}$ and $\mathcal{J}_{i i}$ are given by

$$
\begin{aligned}
\mathcal{J}_{\phi} & =e^{-2 \phi}\left(\ddot{\phi}-\dot{\phi}^{2}+Q e^{\phi} \dot{\phi}\right), \\
\mathcal{J}_{i i} & =2 a^{2}\left(\ddot{\phi}+3 H \dot{\phi}+\dot{\phi}^{2}+(1-q) H^{2}+Q e^{\phi}(\dot{\phi}+H)\right), \quad i=1,2,3,
\end{aligned}
$$

with $q$ the deceleration parameter:

$$
q(z) \equiv-\frac{\ddot{a} a}{\dot{a}^{2}}=-\frac{d H / d t_{E}}{H^{2}\left(t_{E}\right)}-1=\frac{(1+z) d H / d z}{H(z)}-1
$$

For completeness, we mention that the equations (15) lead, after standard manipulations $[8,10]$, to the (non-)conservation equation of matter in the presence of the non-equilibrium contributions:

$$
\frac{d \rho_{m}}{d t_{E}}+3 H\left(\rho_{m}+p_{m}\right)+\frac{1}{2} \frac{d Q}{d t_{E}} \frac{\partial V(\phi)}{\partial Q}-\frac{d \phi}{d t_{E}}\left(\rho_{m}-3 p_{m}\right)=6\left(H+\frac{d \phi}{d t_{E}}\right) a^{-2} \mathcal{J}_{i i}
$$

These equations are used below to obtain a general expression for the Hubble parameter as a function of the redshift, which we use later in our fit to data.

\subsection{Derivation of Basic Phenomenological Formulae for Q-Cosmologies}

The above analysis contains non-linear equations that are hard to solve analytically. A detailed numerical analysis is performed in Ref. [10], where we refer the interested reader for details. Instead, for our phenomenological purposes here, we shall attempt an approximate analytic solution at late epochs of the Universe, appropriate for our phenomenological fits. Throughout this work we work in units where the present scale factor is normalised to one, $a_{0}=1$.

To solve (19) in the various epochs of the Universe, it is convenient first to split the energy density of matter into radiation $\rho_{r}$, baryonic $\rho_{b}$ and dark-matter $\rho_{d}$ components, and to use simple equations of state for the dilaton fluid, as 
supported by our theoretical model [8-10]:

$$
\begin{gathered}
\rho_{m}=\rho_{r}+\rho_{b}+\rho_{d} \equiv \rho_{r}+\rho_{M} \\
p_{b}=0, \quad p_{d}=w_{d} \rho_{d}, \quad p_{r}=\frac{1}{3} \rho_{r}, \quad p_{\phi}=w_{\phi} \rho_{\phi} .
\end{gathered}
$$

We have assumed a non-trivial equation of state $w_{d}$ for the dark matter component, which as we discuss below, stems from the fact that its scaling with the scale factor $a$ is exotic, different from the ordinary dust scaling $a^{-3}$. One can split the matter evolution equation (19) into various components, using (20).

In the solution of Ref. [9] without matter, at late eras the dilaton $\phi$ and the scale factor $a(t)$ vary as follows with the cosmic time $t_{E}$ :

$$
\begin{gathered}
\phi=-\ln a\left(t_{E}\right), \\
a=a_{1}\left(1+\gamma^{2} t_{E}^{2}\right)^{1 / 2},
\end{gathered}
$$

where $a_{1}, \gamma$ are appropriate positive constants. ${ }^{4}$ The form of the scale factor (21) implies the following form for the deceleration parameter at late epochs of the Universe:

$$
q(z)=-1 /\left(\gamma^{2} t_{E}^{2}\right)
$$

The values of the constants are such that the present era in the history of the Universe is characterised by $\gamma t_{E}=\mathcal{O}(1)$, as follows from the fact that the deceleration parameter of the Universe is currently observed $[2 \mathrm{~b}]$ to be $q_{0}=-0.61$.

We notice that the asymptotic behaviour of the model of Ref. [9] without matter, at very late times, indicates that

$$
\dot{\phi}+H \simeq 0
$$

and thus

$$
H \sim a^{-1},
$$

implying that $q \sim 1 / a H$, varying very little at late epochs.

In the present case, where matter effects are important, the formulae need some modification. In general, the dilaton and the scale factor differ from the particular form (21) [10], in which case one obtains a much more complicated behaviour for $q(z)$ than the one given in (22), which can only be studied numerically. A complete numerical analysis is performed in Ref. [10], where we refer the reader for details. We recall, for completeness, that the analysis of

4 In the model of Ref. [9] they are related to the flux in the extra compactified dimensions of the appropriate string theory, but they are viewed here as arbitrary constants in our more general setting. 
Ref. [10] indicates evidence for a past deceleration of the Universe at redshifts larger than $z_{*}=0.37$.

For our generic phenomenological purposes in this work we can make several physically sensible simplifying assumptions, which allow us to carry some of the results of Ref. [9] through to the present case, allowing for some analytic treatment. Specifically, the dilaton and scale factors are assumed to take the form

$$
\phi=-\ln a\left(t_{E}\right)+\mathcal{O}\left(g_{s}^{2}\right)
$$

as dictated by a general analysis of the dilaton equation in the model. ${ }^{5}$ Notice that, in view of the general discussion in Ref. [10], we no longer maintain the explicit form of $a=a_{1}\left(1+\gamma^{2} t_{E}^{2}\right)^{1 / 2}$ used in the matter-less model of Ref. [9], however we do maintain the scaling relation (24), which, according to the numerical analysis of Ref. [10], seems to be a good approximation in the eras of interest to us here.

In general [10], there may be a more complicated behaviour than (23) or (24). For instance, loop and other corrections may yield power-series corrections in the (perturbative) string coupling

$$
\dot{\phi}+H=\mathcal{O}\left(H g_{s}^{2}\right)+\ldots, \quad g_{s}=e^{\phi} .
$$

Such corrections could lead to appreciable deviations in the behaviours of various fields and other cosmological parameters even at the present era. In fact, the numerical analysis of Ref. [10] indicates evidence for a past deceleration of the Universe at redshifts smaller than $z_{*}=0.37$. Nevertheless, even in these more general models, the Hubble parameter maintains [10] a scaling similar to (24) at the late eras of interest to us here, which implies that some of the qualitative features of our analytic approach used here are valid, to a good approximation, in these more complicated cases.

On using (15) and performing some elementary manipulations, we easily obtain:

$$
\rho_{M} \simeq 2(2 q-1) H^{2}-\frac{1}{2} \frac{\partial V}{\partial \phi}+\frac{3}{2} V+\frac{1}{2} e^{2 \phi} \mathcal{J}_{\phi}
$$

A consistent assumption, as we shall demonstrate now, is that the matter energy density, $\rho_{M}$, includes dust (baryonic matter) and exotic scaling (dark) components, which can behave like radiation-like contributions:

$$
\rho_{M} \sim \rho_{\text {dust }}^{0} a^{-3}+\rho_{\text {exotic }}^{0} a^{-4}+\ldots
$$

Indeed, taking into account (24), we observe that the above assumption is consistent with the conservation equation (19) provided that the central charge

\footnotetext{
5 We thank V. Georgalas for an informative discussion on this point.
} 
deficit at late eras scales as

$$
Q^{2}(a) \simeq Q_{*}^{2}+\frac{\rho_{\text {dust }}^{0}}{a}
$$

with $Q_{*}^{2}>0$ a constant, namely the central charge of the asymptotic conformal field theory to which the string model flows at infinite time [8, 9].

Notice that, if one allowed an exotic $a^{-2}$ scaling of matter of the form $\rho_{\phi-\text { like }}^{0} a^{-2}$, which characterises the dilaton dark energy $\rho_{\phi}$, as we discuss below, then $Q^{2}$ should contain also terms of the form $\rho_{\phi-\text { like }}^{0} \ln \left(a^{2}\right)$. This would lead, in general, to logarithmic scaling $a^{-2} \ln (a)$ of the various energy density components, which would complicate the situation, as far as the validity of the approximation (24) is concerned. Although such terms may exist in some models, we do not consider them in this work. Here we assume the scaling (28) for the matter energy densities in the eras of interest to us. For more complete, numerical treatments we refer the interested reader to Ref. [10].

To proceed with a consistency check and estimates on the scaling behaviour of $\rho_{m}, \rho_{\phi}$, it is necessary to make some assumptions about the form of the potential $\tilde{V}$. As already mentioned, we assume that it is generated by higher string loops. In brane-inspired models such as those considered in Refs. [8, 9], one has open strings living on the brane world. The corrections then will be proportional to an extra power of the string coupling $g_{s}=e^{\phi}$. Assuming loop corrections of the form ${ }^{6}$

$$
\tilde{V} \sim \alpha e^{3 \phi}+\beta e^{4 \phi}+\ldots, \quad \alpha, \beta, \ldots=\text { const. },
$$

and taking into account (29), we observe that the dark matter density (27) yields:

$$
\rho_{M} \sim \frac{2(2 q-1)+Q_{*}^{2}}{a^{2}}+\frac{\rho_{\text {dust }}^{0}}{a^{3}}-\frac{\beta}{2} \frac{1}{a^{4}}+\frac{1}{2} e^{2 \phi} \mathcal{J}_{\phi}+\ldots .
$$

As we see from (26), (24), (17), (29), the quantity $\mathcal{J}_{\phi}=\mathcal{O}\left(a^{-2}\right)$ is negative. We also note that the late-era scaling (28), which is required for consistency (on account of (29)), can easily be guaranteed, provided the terms $2(2 q-1)+Q_{*}^{2}$ which otherwise would yield a $a^{-2}$ scaling are suppressed for the redshift region $0<z<2$ of interest to us. This would require a $Q_{*}^{2}$ of order $2\left|\left(2 q_{0}-1\right)\right|$, according to our assumption that $q$ does not change much in the region in which we are interested. ${ }^{7}$

$\overline{6}$ The dots indicate higher-order corrections in the string coupling and also possible dilaton-independent contributions.

7 We remark at this point that the actual data may deviate from this for redshifts less than 0.5, indicating past deceleration of the Universe. However, even in such a case, the absence of $a^{-2}$ terms in matter today might be a consistent assumption. In fact, the numerical solution of Ref. [10] indicates that the matter does develop an exotic scaling $a^{-2}$ but at much later eras than the present one. 
Notice that the dust-like scaling does not receive contributions from the string loop corrections, and in fact a comparison between (31) and (27) yields a mathematically consistent result with $\rho_{\text {dust }}^{0}$ free parameter to be constrained by fitting data and/or other microscopic model considerations.

In contrast, the exotic scaling $a^{-4}$ receives contributions from the loop corrections, proportional to $\beta$, whose sign cannot be fixed by our generic considerations, and depends on the details of the microscopic string/brane theory model, and contributions due to the off-shell Liouville terms $e^{2 \phi} \mathcal{J}_{\phi}$ which are positive according to our assumption that $Q_{*}>0$ :

$$
\rho_{\text {exotic }}^{0} \sim-\frac{\beta}{2}-\frac{|q|+Q_{*}}{2} .
$$

The asymptotic behaviour of the dilaton dark energy may be evaluated from the definition of $\rho_{\phi}(15)$ :

$$
\begin{aligned}
\rho_{\phi} & =\dot{\phi}^{2}+\frac{V}{2} \sim H^{2}+\frac{Q_{*}^{2}}{a^{2}}+\frac{\rho_{\text {dust }}^{0}+\alpha / 2}{a^{3}}+\frac{\beta}{2 a^{4}}+\ldots \\
& =\mathcal{O}\left(a^{-2}\right)+\mathcal{O}\left(a^{-3}\right)+\frac{\beta}{2} a^{-4}+\ldots,
\end{aligned}
$$

where the dots have the same meaning as in (30). It is interesting to observe that the dilaton dark energy component contains a part scaling like $a^{-2}$, which is positive and of order $\left(1+Q_{*}^{2}\right) a^{-2}$, but also parts scaling like $a^{-3}$ and $a^{-4}$, which appear also in the matter energy density (27). However, in contrast to the matter case (31), the 'dust'-like contributions to the dilaton dark energy do not have a fixed sign or magnitude, as they depend on the string loop correction parameter $\alpha$,

$$
\rho_{\phi, 3}^{0} \sim 2 \rho_{\text {dust }}^{0}+\alpha / 2 \sim(1+\alpha) / 2
$$

When combined with the corresponding parts in $\rho_{M}$, in order to obtain the precise scaling of the Hubble parameter with the redshift $z, H(z)$, from the first of equations (15), we observe that

$$
\rho_{\phi}+\rho_{M} \simeq\left|\mathcal{O}\left(a^{-2}\right)\right|+\frac{4 \rho_{\text {dust }}^{0}+\alpha}{2} a^{-3}+\left(-\frac{|q|+Q_{*}}{2}+\ldots\right) a^{-4}
$$

where we have been careful to indicate the relative signs of the various terms, as predicted by the model with the approximations made so far. ${ }^{8}$ Notice the independence of the exotic scaling on the loop parameter $\beta$, which does not enter the expression for $H(z)$. Thus, it is a feature of this Liouville model that

8 One may have deviations from (26) [10], resulting in extra contributions to the $a^{-4}$ scaling, denoted by dots. Thus, the exotic (dark) matter $\Omega_{\delta}$ could be positive. This case seems to be favoured by the data, as discussed in the text, and it is in agreement with the numerical analysis of Ref. [10]. 
the sign of the dust-like contribution appearing in $H(z)$ is not fixed, since such terms receive contributions from the dilaton dark energy. Indeed, in modern string/membrane models, one may obtain negative dust-like contributions to the dilaton potential from compactification of Kaluza-Klein graviton modes in brane models, etc. [17]. In contrast, the exotic scaling terms appear positive.

When fitting the data, in order to distinguish ordinary matter from dilaton dark energy effects that scale similarly with the redshift, one would have to perform also measurements of the equation of state of each component, which we do not attempt in this work. In the specific model [9] considered here as our pilot study, the values of the various $\Omega_{i}$ appearing in the expression for $H(z)$ are in principle predicted by the underlying microscopic dynamics of the model, as we have seen above, but we leave this exercise for future work.

However, since some of the $\Omega_{i}$ involve highly model-dependent coefficients of string loop corrections and other, currently unknown, details of microscopic models, and, moreover, in the above derivation several assumptions have been made, e.g., about the form of the potential for the dilaton, the form of the dilaton and the scale factor at late epochs (21), etc., which may not always be valid [10], we consider at present the various parameters appearing in the expression for $H(z)$ as arbitrary parameters to be fixed by the data. To be even more general, we keep the exponent of the exotic scaling as a free fitting parameter as well. From the above considerations, in particular (28), (33), it is also clear that in general $\Omega_{\text {dust }}$ may be negative, as its sign depends on the sign of the corrections (30) to the dilaton potential.

The final result of our parametrisation for $H(z)$ in the Q-cosmology framework, which will be compared with the astrophysical data in the next section, in order to obtain information on the basic cosmological parameters of the model, is therefore:

$$
H(z)=H_{0}\left(\Omega_{3}(1+z)^{3}+\Omega_{\delta}(1+z)^{\delta}+\Omega_{2}(1+z)^{2}\right)^{1 / 2},
$$

with the densities $\Omega_{2,3, \delta}$ corresponding to present-day values $(z=0)$ and

$$
\Omega_{3}+\Omega_{\delta}+\Omega_{2}=1
$$

The reader should recall that in the model described in this section $\delta=4$. However, in view of the various approximations employed in our analytic treatment of $Q$-cosmology above, which, as already mentioned, may not be valid in the present era, when matter contributions are important, the exponent $\delta$ is treated from now on as a fitting parameter. The above formulae are valid for late eras, such as the ones pertinent to the supernova and other data $(0 \leq z \leq 2)$ that we use in this work.

We stress once again that the various $\Omega_{i}$ contain contributions from both dark energy and matter energy densities. As explained previously, $\Omega_{3}$ does not 
merely represent ordinary matter effects, but also receives contributions from the dilaton dark energy. In fact, the sign of $\Omega_{3}$ depends on details of the underlying theory, and it could even be negative. For instance, Kaluza-Klein graviton modes in certain brane-inspired models [17] yield negative dust contributions. In a similar vein, the exotic contributions scaling as $(1+z)^{\delta}$ are affected by the off-shell Liouville terms of Q-cosmology. It is because of the similar scaling behaviours of dark matter and dilaton dark energy that we reverted to the notation $\Omega_{i}, i=2,3, \delta$ in (36). To disentangle the ordinary matter and dilaton contributions one may have to resort to further studies on the equation of state of the various components, which we do not study in this article. More generally, one could have included a cosmological constant $\Omega_{\Lambda}$ contribution in (36), which may be induced in certain brane-world inspired models. We do not do so in this work, as our primary interest is to fit Q-cosmology models [8-10], which are characterised by dark energy densities that relax to zero.

\section{Data Analysis}

\subsection{Astrophysical Observables}

We now discuss the observables available to test the above models using mainly data on high-redshift supernovae and (to a limited extent) baryon acoustic oscillations.

We use the supernovae data reported in Refs. [4, 18], which are given in terms of the distance modulus

$$
\mu=5 \log d_{L}+25 .
$$

We use for $d_{L}$ the standard formula relating the luminosity distance to the redshift $z$

$$
d_{L}=c(1+z) \int_{0}^{z} \frac{d z^{\prime}}{H\left(z^{\prime}\right)}
$$

where $H$ is given by (2),(6) and (36) in the three models discussed in the previous sections, namely $\Lambda$ CDM, super-horizon and Q-cosmology respectively. We note that this observable depends on the expansion history of the Universe from $z$ to the present epoch, and recall that most of the available supernovae have $z<1$, though there is a handful with larger values of $z$.

For standard on-shell cosmologies, complementary information is provided by the data on baryon acoustic oscillations (BAO) $[19,20]$, which show up in the galaxy-galaxy correlation function at $z \sim 0.35$. However, for reasons to be discussed later on in the article, the application of such an analysis to the off-shell Q-cosmology model is an open issue, since the underlying theoretical framework needs to be re-evaluated.

The formulae for $H(z)$ in the various models discussed above, namely (2), (6) and (36), serve as the basis for our analysis below. 


\subsection{Experimental Fits}

We now present our fits of the above models to the presently available astrophysical data on high-redshift supernovae [4, 18], and (in the case of $\Lambda$ CDM) also baryon acoustic oscillations (BAO) [19, 20]. We start our discussion with the $\Lambda$ CDM and Super-Hubble-Horizon models, and then proceed to the case of off-shell Liouville Q-cosmology models [8-10].

Measurements [1, 4] are available of the distance moduli (38) for 157 supernovae in a so-called 'gold' sample [4], observed by ground-based facilities and the Hubble Space Telescope: see Fig. 1. We compare these with the measurements of 71 other high-redshift supernovae published more recently [18] by the Supernova Legacy Survey (SNLS), which are accompanied by a reference sample of 44 nearby SN Ia, yielding a total of 115 data points: see Fig. 2. For both samples, the data are expressed in terms of the observed distance modulus $\mu_{o b}$ derived from the SN Ia light curves and the corresponding redshift $z$. The best-fit cosmological parameters are then extracted by minimizing:

$$
\chi^{2}=\sum_{i} \frac{\left(\mu_{o b, i}-\mu_{t h}\left(z_{i}\right)\right)^{2}}{\sigma_{o b, i}^{2}+\sigma_{i n t}^{2}},
$$

where $\mu_{o b, i}$ and $\sigma_{o b, i}$ are the measured value for the distance modulus and the corresponding error for a specific supernova $i$, respectively, $\sigma_{\text {int }}$ is the intrinsic dispersion of the absolute magnitudes of the supernovae, and $\mu_{t h}\left(z_{i}\right)$ is the theoretical prediction for a given model as calculated by (38), (39), where $d_{L}$ is given in units of megaparsecs. This fit and all subsequent analyses are performed with the ROOT [21] implementation of the Minuit function minimization and error analysis code [22].

Both the data and the predictions of the various models are expressed in the following as residuals, $\Delta \mu$, from their predicted values in an empty Universe $\left(\Omega_{\mathrm{M}}=0\right)$ :

$$
d_{L}^{(e m p t y)}=\frac{c}{2 H_{0}} z(2+z) .
$$

In addition to presenting the 'gold' and the SNLS data sets respectively, Figures 1 and 2 also display the predictions of various cosmological models to be detailed later on in the article.

In addition to the $157 \mathrm{SN}$ Ia belonging to the 'gold' sample analysed in Ref. [4], a so-called 'silver' dataset of $29 \mathrm{SN} 1 \mathrm{a}$ is also available, with lesser spectrometric and photometric quality compared to the 'gold' one. Although the results presented here were obtained with the 'gold' dataset, the analysis was also repeated including the 'silver' supernovae data — with a total of 186 supernovae - with results comparable to those presented here, proving the robustness of the analysis. The analysis was also repeated for the combined sample of the 'gold' (157 supernovae) plus the SNLS sample (71 supernovae), which yields a sample of 228 supernovae. 


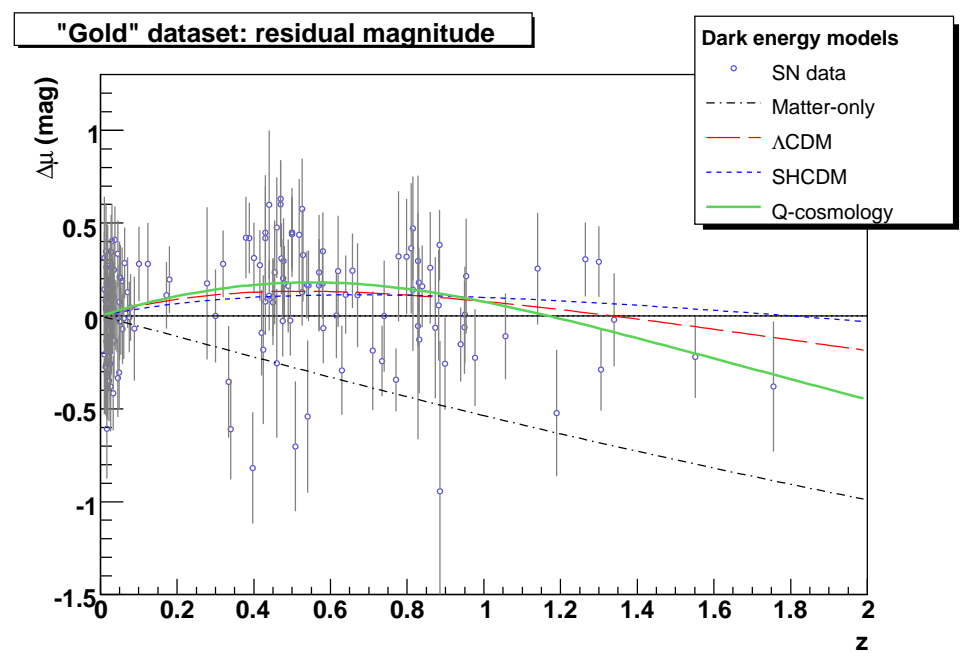

Fig. 1. Residual magnitude of the 'gold' dataset supernovae as a function of the redshift for the best-fit values of the cosmological parameters. The following models are shown: (i) Empty Universe (black solid line); (ii) Universe with matter only, $\Omega_{\mathrm{M}}=1$ (black dashed-dotted line); (iii) $\Lambda$ CDM model for $\Omega_{\mathrm{M}}=0.287$ (red long-dashed line); (iv) the super-horizon model for $\Psi_{\ell 0}=-0.79$ (blue short-dashed line); and (v) off-shell Q-cosmology model for $\Omega_{3}=-3.6$ and $\Omega_{4}=1.2$ (green thick solid line).

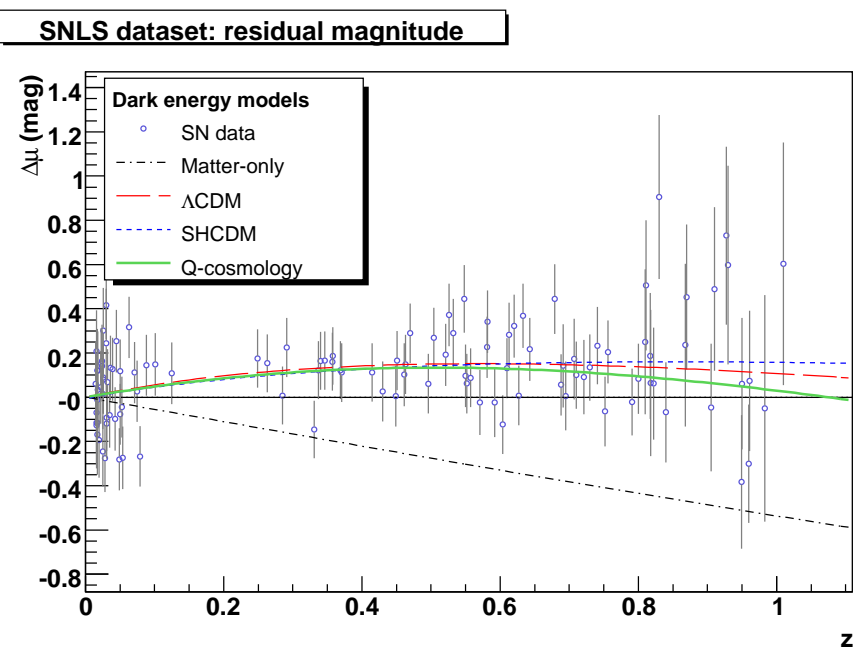

Fig. 2. Residual magnitude of the SNLS dataset supernovae as a function of the redshift for the best-fit values of the cosmological parameters. The following models are shown: (i) Empty Universe (black solid line); (ii) Universe with matter only $\Omega_{\mathrm{M}}=1$ (black dashed-dotted line); (iii) $\Lambda$ CDM model for $\Omega_{\mathrm{M}}=0.265$ (red long-dashed line); (iv) the super-horizon model for $\Psi_{\ell 0}=-0.94$ (blue short-dashed line); and (v) off-shell Q-cosmology model for $\Omega_{3}=-3.0$ and $\Omega_{4}=1.0$ (green thick solid line).

The $\chi^{2}$ values obtained for the best fits to the various models are discussed in the remainder of this Section. 

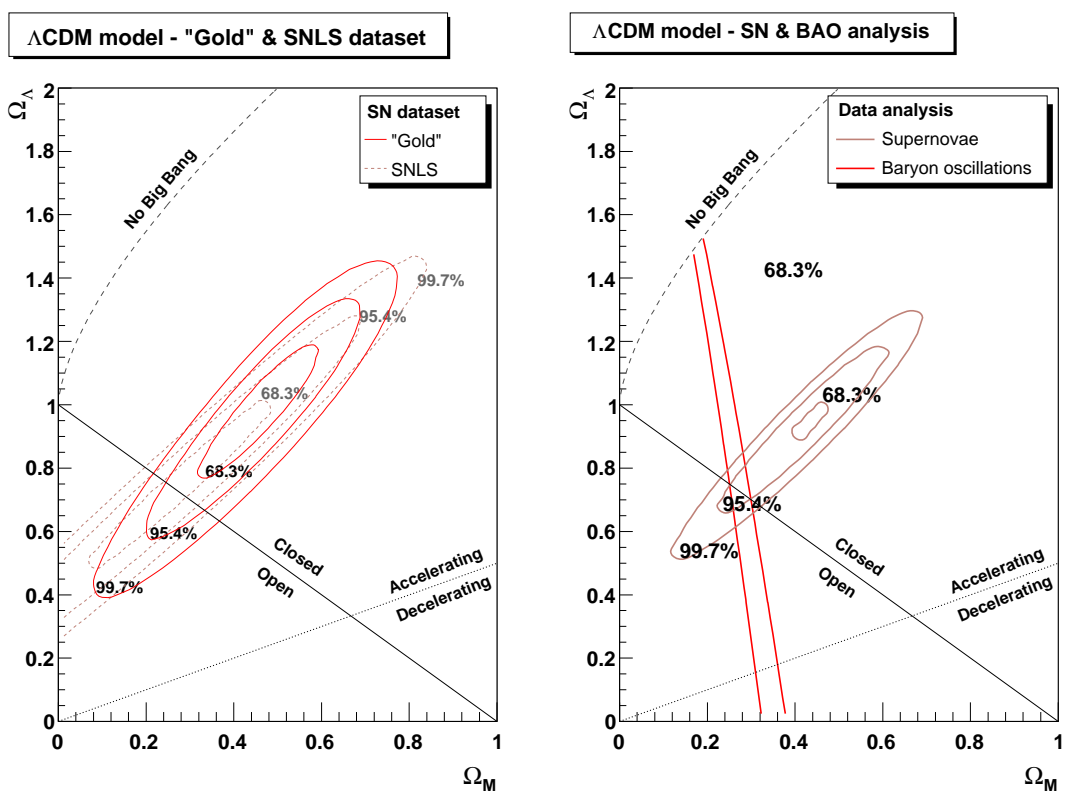

Fig. 3. Confidence-level contour plots for the $\Lambda \mathrm{CDM}$ model. (i) Left panel: 'gold' sample of 157 supernovae [4] and SNLS sample of $71+44$ supernovae [18], (ii) right panel: combined 'gold' and SNLS sample of $157+71$ supernovae. The $68.3 \%$ C.L. region extracted from the $\mathrm{BAO}$ analysis is also superimposed.

\subsubsection{Cosmological-constant model}

For the $\Lambda$ CDM model [5], assuming a flat Universe, we find the $\chi^{2}$ and parameter values listed in Table 1 , which are in agreement with those presented in Ref. $[4,18]$. The corresponding confidence limits in the $\left(\Omega_{\mathrm{M}}, \Omega_{\Lambda}\right)$ plane are shown in Fig. 3.

Table 1

Fits to the $\Lambda$ CDM model parameter $\Omega_{\mathrm{M}}$, assuming a flat Universe. We compare the values favored by the 'gold' and the SNLS data sets, and give in the third row the results of a fit to the combined data set.

\begin{tabular}{llll}
\hline SN data set & $\Omega_{\mathrm{M}}$ & $\chi^{2}$ & $\chi^{2} /$ dof \\
\hline 'gold' & $0.287 \pm 0.026$ & 178 & 1.14 \\
SNLS & $0.265 \pm 0.022$ & 114 & 1.00 \\
Combined & $0.274 \pm 0.017$ & 239 & 1.05 \\
\hline
\end{tabular}

We see from Table 1 and Fig. 3 that the fits to the two data sets are quite compatible. It is therefore reasonable to make a joint fit to the combined data set, which yields the results shown in the third row of Table 1: the low value of the $\chi^{2} /$ dof justifies this combination a posteriori. For the value of $\Omega_{\mathrm{M}}$ found there, the critical redshift $z_{*}$ above which deceleration occurs is $z_{*} \simeq 0.74$. 

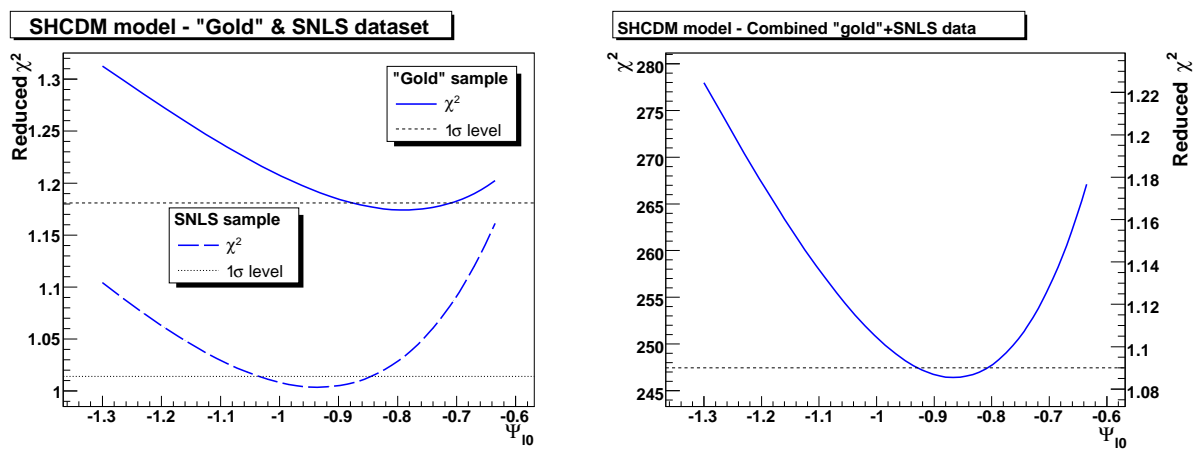

Fig. 4. The likelihood function $\chi^{2}$ and the reduced $\chi^{2}$ per degree of freedom as a function of $\Psi_{\ell 0}$ of the super-horizon model [6]. (i) Left figure: 'Gold' sample of 157 supernovae [4] and SNLS sample of $71+44$ supernovae [18]; (ii) right figure: combined 'gold' and SNLS sample of $157+71$ supernovae.

\subsubsection{Super-horizon model}

For the super-horizon model of Ref. [6] we find the fit values shown in Table 2. Figure 4 displays the $\chi^{2}$ values for the two data sets, as functions of the model parameter $\Psi_{\ell 0}$. In this case, the consistency between the fits to the 'gold' and SNLS data sets is not as good as for the $\Lambda$ CDM model. Nevertheless, the overall fit quality is acceptable, and the third line of Table 2 displays the parameter values found in a combined fit: the low value of the $\chi^{2} /$ dof again justifies this combination a posteriori. In this model, the critical redshift is $z_{*}=4.6$.

Table 2

Fits to the SHCDM model [6] parameter $\Psi_{\ell 0}$ using the 'gold' and the SNLS data sets and, in the third line, the result of a fit to the combined data set.

\begin{tabular}{llll}
\hline SN data set & $\Psi_{\ell 0}$ & $\chi^{2}$ & $\chi^{2} /$ dof \\
\hline 'gold' & $-0.79 \pm 0.08$ & 183 & 1.17 \\
SNLS & $-0.94 \pm 0.09$ & 114 & 1.00 \\
Combined & $-0.87 \pm 0.06$ & 245 & 1.09 \\
\hline
\end{tabular}

The value of the critical redshift $z_{*}$ marking the transition from deceleration to acceleration may be compared in each of the above cases with that reported in Ref. [4]. We note that the precise value of $z_{*}$ depends crucially on the microscopic model used, in particular on the equation of state, which is in general $z$-dependent. This affects the precise functional dependence on $z$ of the Hubble parameter $H(z)$ and the deceleration parameter $q(z)$, as seen in Fig. 5. The analysis of Ref. [4], which indicated a critical $z_{*}=0.46 \pm 0.13$, was based on a very simple assumed form (linear) for the $z$-dependent deceleration rate, namely $q(z)=q_{0}+q_{1} z$, which is not the case in all models, as seen in the right panel of Fig. 5. We therefore consider that the issue of the exact value of $z_{*}$ is a delicate point to be explored in the future. 

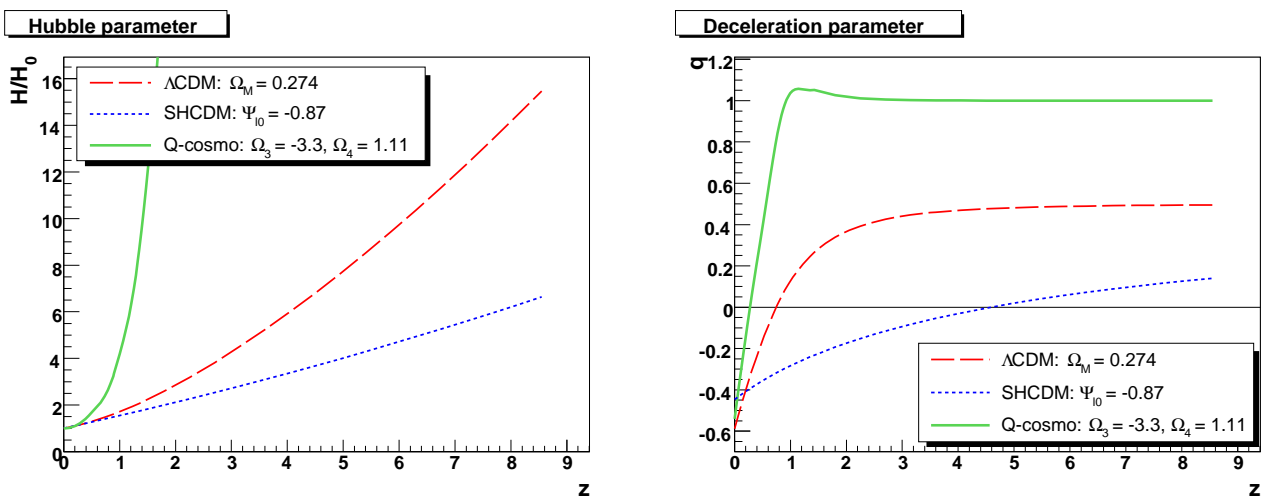

Fig. 5. The Hubble parameter (left) and the deceleration parameter (right) as a function of the redshift $z$ for various cosmological models: (i) red long dashed curve, the standard $\Lambda$ CDM model, (ii) blue short dashed curve, the SHCDM model of Ref. [6], and (iii) green solid curve, Q-cosmology (off-shell) model with dark energy relaxing as $1 / t^{2}$.

\subsubsection{Q-Cosmology (off-shell) Model}

We now present a detailed fit of the off-shell Q-cosmology model described above to the presently available astrophysical data on supernovae $[4,18]$, checking the consistency of the model and determining the crucial cosmological parameters of this generic class of Liouville Q-cosmology models of Refs. [8-10].

We again analyze the supernovae data $[4,18]$, as given in terms of the distance modulus. We use the formula (39), with the Hubble parameter given by (36), to obtain $d_{L}$ as predicted by the model of Refs. [8, 9]. As before, we use the 157 data points from the so-called 'gold' sample of supernovae [1, 4] observed by ground-based facilities and Hubble Space Telescope, as well as the more recent SNLS sample of 115 supernonae reported in Ref. [18]. The best-fit parameter values and the corresponding values for the likelihood function $\chi^{2}$ are listed in Tables 3 and 4 for both datasets and their combination.

The analysis in Table 3 has three free parameters, namely $\Omega_{3}, \Omega_{\delta}$ and the exponent $\delta$. As in previous fits, the parameter values favoured by the 'gold' and SNLS supernovae samples are quite compatible, so we concentrate on fit to the combined data. We see that the three contributions to the energy density, $\Omega_{2,3, \delta}$, are not very well determined individually, but the fit prefers a value close to 4 for the exponent $\delta$. We therefore repeated the fit, this time fixing $\delta=4$, with the results shown in Table 4 . The quality of the combined fit is no worse than with $\delta$ left free, and the values of the different energy densities $\Omega_{2,3,4}$ are compatible with those in Table 3 , but with significantly smaller errors. The Q-cosmology curves in Figs. 1 and 2 are based on the best-fit values in Table $4 .{ }^{9}$ Off-shell contributions to the 'dust' term $\Omega_{3}$ allow

$\overline{9}$ This analysis was repeated using also the so-called 'silver' supernovae, and the 
it to take negative values, so the negative signs found in the fits in Tables 3 , 4 are quite consistent with this framework.

Table 3

Q-cosmology model parameter values favoured by the 'gold' and the SNLS supernovae datasets. $\Omega_{2}$ is determined by the other densities so that $\Omega_{\delta}+\Omega_{3}+\Omega_{2}=1$.

\begin{tabular}{lllllll}
\hline SN dataset & $\Omega_{3}$ & $\Omega_{\delta}$ & $\delta$ & $\Omega_{2}$ & $\chi^{2}$ & $\chi^{2} /$ dof \\
\hline 'Gold' & $-3.2 \pm 0.4$ & $1.0 \pm 1.7$ & $4.10 \pm 0.20$ & $3.2 \pm 1.7$ & 177 & 1.14 \\
SNLS & $-2.0 \pm 0.8$ & $0.4 \pm 0.4$ & $4.7 \pm 1.0$ & $2.6 \pm 0.9$ & 113 & 1.01 \\
Combined & $-3.7 \pm 1.1$ & $1.3 \pm 0.7$ & $3.9 \pm 0.3$ & $3.4 \pm 1.3$ & 237 & 1.05 \\
\hline
\end{tabular}

Table 4

Q-cosmology model parameter values favoured by the 'gold' and the SNLS supernovae datasets for a fixed value $\delta=4 . \Omega_{2}$ is determined by the other densities so that $\Omega_{4}+\Omega_{3}+\Omega_{2}=1$.

\begin{tabular}{llllll}
\hline SN dataset & $\Omega_{3}$ & $\Omega_{4}$ & $\Omega_{2}$ & $\chi^{2}$ & $\chi^{2} /$ dof \\
\hline 'Gold' & $-3.6 \pm 0.8$ & $1.2 \pm 0.3$ & $3.4 \pm 0.9$ & 177 & 1.14 \\
SNLS & $-3.0 \pm 0.9$ & $1.0 \pm 0.4$ & $3.0 \pm 1.0$ & 113 & 1.00 \\
Combined & $-3.3 \pm 0.6$ & $1.11 \pm 0.25$ & $3.2 \pm 0.7$ & 237 & 1.05 \\
\hline
\end{tabular}

We observe from Fig. 5 that, with the best-fit values of the Q-cosmology parameters in the model of Refs. [8, 10], the transition from a decelerating expansion of the Universe to an accelerating expansion would have taken place when $z=z_{*} \simeq 0.31$. This is almost compatible at the $1-\sigma$ level with the result of the analysis of Ref. [4], which indicated a critical $z_{*} \simeq 0.46 \pm 0.13$. However, this was based on a very simple (linear) assumed form for the deceleration $q(z)=q_{0}+q_{1} z$, which is not the case of our model, as also seen in Fig. 5. It should be possible in the future to explore observationally nonlinear forms of $w_{\phi}(z)$, with the aim of improving the fit and reducing the uncertainty in the value of the critical $z=z_{*}$ for the transition from deceleration to acceleration.

\section{Results and Outlook}

We have shown in this paper that the two main data sets for high-redshift supernovae are compatible, at least as far as their constraints on the cosmological models studied in this paper are concerned. The standard $\Lambda$ CDM model fits the supernova data very well, and the super-horizon dark matter model also fits the supernova data quite well. Both of these models are on-shell, i.e., they satisfy the pertinent Einstein's equations. However, off-shell cosmology models

results were comparable to the ones presented here, confirming the robustness of our analysis. 
can still be compatible with the data. As we discussed above, off-shell effects are important in our Q-cosmology model. Introducing an extra parameter to allow for these off-shell effects, we find that the $Q$-cosmology model may fit the supernovae data as well as the standard $\Lambda C D M$ model.

The best fits acquired by combining the 'gold' sample [4] and the SNLS [18] supernovae data set have the following $\chi^{2}$ values:

- In the $\Lambda$ CDM model that combines a cosmological constant with cold dark matter, assuming a flat Universe we find $\chi^{2}=239\left(\chi^{2} /\right.$ dof $\left.=1.05\right)$ for $\Omega_{\mathrm{M}}=0.274 \pm 0.017$, corresponding to a value of $\Omega_{\Lambda}=0.726 \pm 0.017$ for the dark energy density. This value is consistent with the one predicted in Ref. [4].

- In the super-horizon dark matter model (SHCDM) of Ref. [6], we find $\chi^{2}=$ $245\left(\chi^{2} /\right.$ dof $\left.=1.09\right)$ for the best-fit case with $\Psi_{\ell 0}=-0.87 \pm 0.06$.

- For the Q-cosmology model of Refs. [8, 10], assuming $\delta=4: \chi^{2}=237$ $\left(\chi^{2} /\right.$ dof $\left.=1.05\right):$ for $\Omega_{3}=-3.3 \pm 0.6, \Omega_{4}=1.11 \pm 0.25$, yielding $\Omega_{2}=$ $3.2 \pm 0.7$.

Just as this analysis was being completed, the three-year WMAP data (WMAP3) were released [2b]. Assuming a flat Universe, the WMAP3 data by themselves yield within the $\Lambda$ CDM model the result $\Omega_{\mathrm{M}}=0.238_{-0.041}^{+0.030}$. This central value is slightly lower than our result, but the error is larger, and the two results are compatible within their errors. The WMAP collaboration has combined their data with other CMB data, and the most precise result, obtained by combining WMAP3 with the $2 \mathrm{dF}$ Galaxy Redshift Survey, is $\Omega_{\mathrm{M}}=0.236_{-0.024}^{+0.016}$, which differs from our combined result by about $2 \sigma$. On the other hand, combining WMAP3 with large-scale structure data gives larger central values of $\Omega_{\mathrm{M}}$. The smallest errors are those obtained by combining WMAP3 with the Sloan Digital Sky Survey LRG data set: $\Omega_{\mathrm{M}}=0.267_{-0.025}^{+0.018}$ and with the CFHTLS lensing data: $\Omega_{\mathrm{M}}=0.299_{-0.025}^{+0.019}$. These results straddle our combined fit to the supernova data.

The data on baryon acoustic oscillations (BAO) provide complementary information [19, 20]. Within the $\Lambda$ CDM model and assuming a flat Universe, the BAO yield an independent estimate $[20] \Omega_{\mathrm{M}}=0.273 \pm 0.024$, which is completely compatible with the value we obtained from the supernova data. Combining the two results, we find $\Omega_{\mathrm{M}}=0.274 \pm 0.014$. We leave for a future occasion the exploration of the constraints imposed by the BAO data on the Q-cosmology models. When confronting the BAO data with the Q-cosmology model, it will be desirable also to formulate more precisely the predictions of this model, which is also an important subject for future work. There is one important issue which should be stressed at this point. The exotic matter scaling: $a^{-\delta}$ with $\delta \sim 4$ of the Q-cosmology model at late eras, if valid at earlier times, would have dominated the epochs characterised by redshifts $z>10$. As a result, the positions of the peaks of the Baryon Acoustic Oscillations would 
have been modified, leading to stringent constraints on the models. However, in such a scenario the phenomenological observables currently used in analyses of the BAO [20] would be inapplicable to Q-cosmology models. We hope to come back to this issue in a future publication.

\section{Acknowledgements}

N.E.M. wishes to thank G. Diamandis, V. Georgalas and A. B. Lahanas for discussions, and the Physics Department of Athens University for its hospitality during the last stages of this work. The work of N.E.M. is partially supported by funds made available by the European Social Fund (75\%) and National (Greek) Resources (25\%) - EPEAEK B - PYTHAGORAS. V.A.M. acknowledges support by the European Union through the RTN contract: HPRN-CT-2002-00292, The Third Generation as a Probe for New Physics. The work of D.V.N. is supported by D.O.E. grant DE-FG03-95-ER-40917.

\section{References}

[1] A. G. Riess et al. [Supernova Search Team Collaboration], Astron. J. 116, 1009 (1998) [arXiv:astro-ph/9805201]; B. P. Schmidt et al. [Supernova Search Team Collaboration], Astrophys. J. 507, 46 (1998) [arXiv:astroph/9805200]; S. Perlmutter et al. [Supernova Cosmology Project Collaboration], Astrophys. J. 517, 565 (1999) [arXiv:astro-ph/9812133]; J. P. Blakeslee et al. [Supernova Search Team Collaboration], Astrophys. J. 589, 693 (2003) [arXiv:astro-ph/0302402]; A. G. Riess et al. [Supernova Search Team Collaboration], Astrophys. J. 560, 49 (2001) [arXiv:astro$\mathrm{ph} / 0104455]$.

[2a] D. N. Spergel et al. [WMAP Collaboration], Astrophys. J. Suppl. 148, 175 (2003) [arXiv:astro-ph/0302209].

[2b] D. N. Spergel et al., arXiv:astro-ph/0603449.

[3] A. Upadhye, M. Ishak and P. J. Steinhardt, Phys. Rev. D 72 (2005) 063501 [arXiv:astro-ph/0411803].

[4] A. G. Riess et al. [Supernova Search Team Collaboration], Astrophys. J. 607, 665 (2004) [arXiv:astro-ph/0402512].

[5] N. A. Bahcall, J. P. Ostriker, S. Perlmutter and P. J. Steinhardt, Science 284, 1481 (1999) [arXiv:astro-ph/9906463].

[6] E. W. Kolb, S. Matarrese, A. Notari and A. Riotto, arXiv:hepth/0503117; E. W. Kolb, S. Matarrese and A. Riotto, arXiv:astro$\mathrm{ph} / 0506534$.

[7] I. Antoniadis, C. Bachas, J. R. Ellis and D. V. Nanopoulos, Phys. Lett. B 211, 393 (1988); Nucl. Phys. B 328, 117 (1989); Phys. Lett. B 257, 278 (1991).

[8] J. R. Ellis, N. E. Mavromatos and D. V. Nanopoulos, Phys. Lett. B 619, 17 (2005) [arXiv:hep-th/0412240]; J. R. Ellis, N. E. Mavromatos, 
D. V. Nanopoulos and M. Westmuckett, Int. J. Mod. Phys. A 21 (2006) 1379 [arXiv:gr-qc/0508105].

[9] G. A. Diamandis, B. C. Georgalas, N. E. Mavromatos and E. Papantonopoulos, Int. J. Mod. Phys. A 17, 4567 (2002) [arXiv:hepth/0203241]; G. A. Diamandis, B. C. Georgalas, N. E. Mavromatos, E. Papantonopoulos and I. Pappa, Int. J. Mod. Phys. A 17, 2241 (2002) [arXiv:hep-th/0107124].

[10] G. A. Diamandis, B. C. Georgalas, A. B. Lahanas, N. E. Mavromatos and D. V. Nanopoulos, arXiv:hep-th/0605181, Phys. Lett. B, in press (doi:10.1016/j.physletb.2006.09.035).

[11] M.B. Green, J.H. Schwarz and E. Witten, Superstring Theory, Vols. I \& II (Cambridge University Press, 1987).

[12] J. Polchinski, String theory, Vols. I \& II (Cambridge University Press, 1998).

[13] F. David, Mod. Phys. Lett. A 3, 1651 (1988); J. Distler and H. Kawai, Nucl. Phys. B 321, 509 (1989); J. Distler, Z. Hlousek and H. Kawai, Int. J. Mod. Phys. A 5, 391 (1990); see also: N. E. Mavromatos and J. L. Miramontes, Mod. Phys. Lett. A 4, 1847 (1989); E. D'Hoker and P. S. Kurzepa, Mod. Phys. Lett. A 5, 1411 (1990).

[14] J. R. Ellis, N. E. Mavromatos and D. V. Nanopoulos, Phys. Lett. B 293, 37 (1992) [arXiv:hep-th/9207103]; Mod. Phys. Lett. A 10, 1685 (1995) [arXiv:hep-th/9503162]. Invited review for the special Issue of J. Chaos Solitons Fractals, Vol. 10, (eds. C. Castro amd M.S. El Naschie, Elsevier Science, Pergamon 1999) 345 [arXiv:hep-th/9805120];

[15] M. Gasperini, F. Piazza and G. Veneziano, Phys. Rev. D 65, 023508 (2002) [arXiv:gr-qc/0108016].

[16] G. Curci and G. Paffuti, Nucl. Phys. B 286, 399 (1987).

[17] M. Minamitsuji, M. Sasaki and D. Langlois, Phys. Rev. D 71, 084019 (2005) [arXiv:gr-qc/0501086].

[18] P. Astier et al., Astron. Astrophys. 447 (2006) 31 [arXiv:astro$\mathrm{ph} / 0510447]$.

[19] For a critical comprehensive recent review see: E. V. Linder, arXiv:astro$\mathrm{ph} / 0507308$ and references therein.

[20] D. J. Eisenstein et al., Astrophys. J. 633 (2005) 560 [arXiv:astroph/0501171]; S. Cole et al. [The 2dFGRS Collaboration], Mon. Not. Roy. Astron. Soc. 362, 505 (2005) [arXiv:astro-ph/0501174].

[21] R. Brun and F. Rademakers, Nucl. Instrum. Meth. A 389 (1997) 81.

[22] F. James and M. Roos, Comput. Phys. Commun. 10 (1975) 343. 